Environnement urbain

Urban Environment

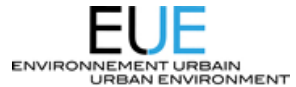

\title{
Architectures émergentes à El Alto (Bolivie): essai d'interprétation sociologique
}

\section{Franck Poupeau}

Volume 9, 2015

URI : https://id.erudit.org/iderudit/1036222ar

DOI : https://doi.org/10.7202/1036222ar

Aller au sommaire du numéro

\section{Éditeur(s)}

Institut National de Recherche Scientifique Centre Urbanisation Culture et Société

ISSN

1916-4645 (numérique)

Découvrir la revue

Citer cet article

Poupeau, F. (2015). Architectures émergentes à El Alto (Bolivie): essai d'interprétation sociologique. Environnement urbain / Urban Environment, 9. https://doi.org/10.7202/1036222ar

\section{Résumé de l'article}

This article applies Bourdieu's analysis of the formation of the "scholastic habitus" in medieval times, elaborated in his 1967 afterword to his French translation of Erwin Panofsky's Gothic Architecture and Scholasticism (2005 [1967]), to a different historical case and social context, namely the correspondence between indigenous mental categories and architectural efflorescence in a city of the contemporary Andes: El Alto. This same principle of homology between mental categories and building layout (rooted in a common habitus) can be used to interpret one of the most spectacular characteristics of "emerging architectures" known as chalets in Bolivia. The term chalet designates a hybrid structure consisting of a penthouse and storey dwelling built on the rooftops. The chalets are architectural forms embedded within an economy of symbolic goods characterized by a "dual truth": they are at once material and symbolic; they perform economic functions while at the same time seeking conspicuous exposure and public visibility. The hypothesis developed here is that the conspicuous lifestyle revealed by the construction of chalets on the rooftops can be understood by reference to the emerging social power of the indigenous (cholos) elites. This article thus identifies in the fraternities of El Alto the structural equivalent of the scholastic institution that Bourdieu associated with Gothic architecture, in order to reveal the formation of a specific habitus, shared among urban categories defined by similar residential locations, economic activities and forms of collective organization.
Tous droits réservés @ Institut National de Recherche Scientifique Centre Urbanisation Culture et Société, 2015
Ce document est protégé par la loi sur le droit d'auteur. L'utilisation des services d'Érudit (y compris la reproduction) est assujettie à sa politique d'utilisation que vous pouvez consulter en ligne.

https://apropos.erudit.org/fr/usagers/politique-dutilisation/ 


\section{Franck Poupeau}

\section{Architectures émergentes à El Alto (Bolivie): essai d'interprétation sociologique}

\section{Introduction}

L'anthropologie des sociétés andines s'est intéressée aux significations sociales attachées à l'habitat et aux relations entre les villes et les campagnes (Kingman, 1992 ; Arnold, 1992), en attirant l'attention sur leurs formes architecturales spécifiques : la construction de bâtiments à plusieurs étages, dotés de fonctions spécifiques, avec des ateliers en rez-de-chaussée, une salle pour événements festifs aux premiers étages, et enfin des logements au dessus. Désormais, c'est la construction d'édifices colorés, surmontés par de petites maisons à part entière sur le toit, entourées de murs imitant les clôtures que l'on trouve dans les campagnes environnantes, et dénommées «chalets », qui attire l'attention (Cárdenas, 2010): en dehors de la sphère académique, on ne compte plus les articles consacrés à ces « architectures » émergentes dans la presse (Neuman, 2013, Mansilla, 2015; Mesa, 2015) ; un documentaire cinématographique y est consacré (UNF, 2015), un livre d'art architectural édité (Andreoli, D'Andrea, 2014) et une visite touristique est même désormais organisée par une agence de voyage. Les propriétaires de ces constructions appartiennent à ce qui est désormais appelé la «bourgeoisie chola» - ces entrepreneurs urbains d'origine aymara ${ }^{1}$, propriétaires de petites affaires familiales qui constituent le cœur de l'activité productive de El Alto (Garfias \& Mazurek, 2005). Le dernier étage surajouté porte le nom de «chalet » [prononcer \{châle-t $\}$ ], en référence directe et distinctive aux domiciles supposés des gens fortunés qui se réfugient, de l'autre côté de l'Atlantique, dans des lieux cossus au cœur des montagnes suisses.

Cette intrusion d'éléments apparemment « étrangers » dans une culture urbaine supposée «autochtone » et « originaire » de la ville de El Alto, devenue l'épicentre des mobilisations « indigénistes » de la Bolivie contemporaine depuis le début des années 2000 (Lazar, 2008), peut paraitre paradoxal et incongru. L'effet de surprise initial peut cependant être reformulé en interrogation plus sociologique, conformément à l'intuition de Robert Darnton selon lequel « les points qui paraissent les plus obscurs sont ceux par lesquels on accède le mieux à la connaissance d'une culture étrangère. Quand nous comprenons que quelque chose nous échappe, une plaisanterie, une cérémonie, un proverbe particulièrement significatif pour les habitants du pays, nous sentons que c'est par là qu'il faut aborder un système de valeurs inconnu pour le démêler » (Darnton, 2011 : 112). Et l'on peut en effet se demander ce qu'il y a encore de véritablement « andin » dans ces « chalets » sur le toit des immeubles, en rupture avec l'image traditionnelle des résidences populaires, principalement indigènes, de l'altiplano. Les couleurs, la multiplication des formes, ne cessent d'interpeler, comme le montre la photographie 1 de l'un des édifices emblématiques des « chalets » de El Alto, sur une place du district 3 . 


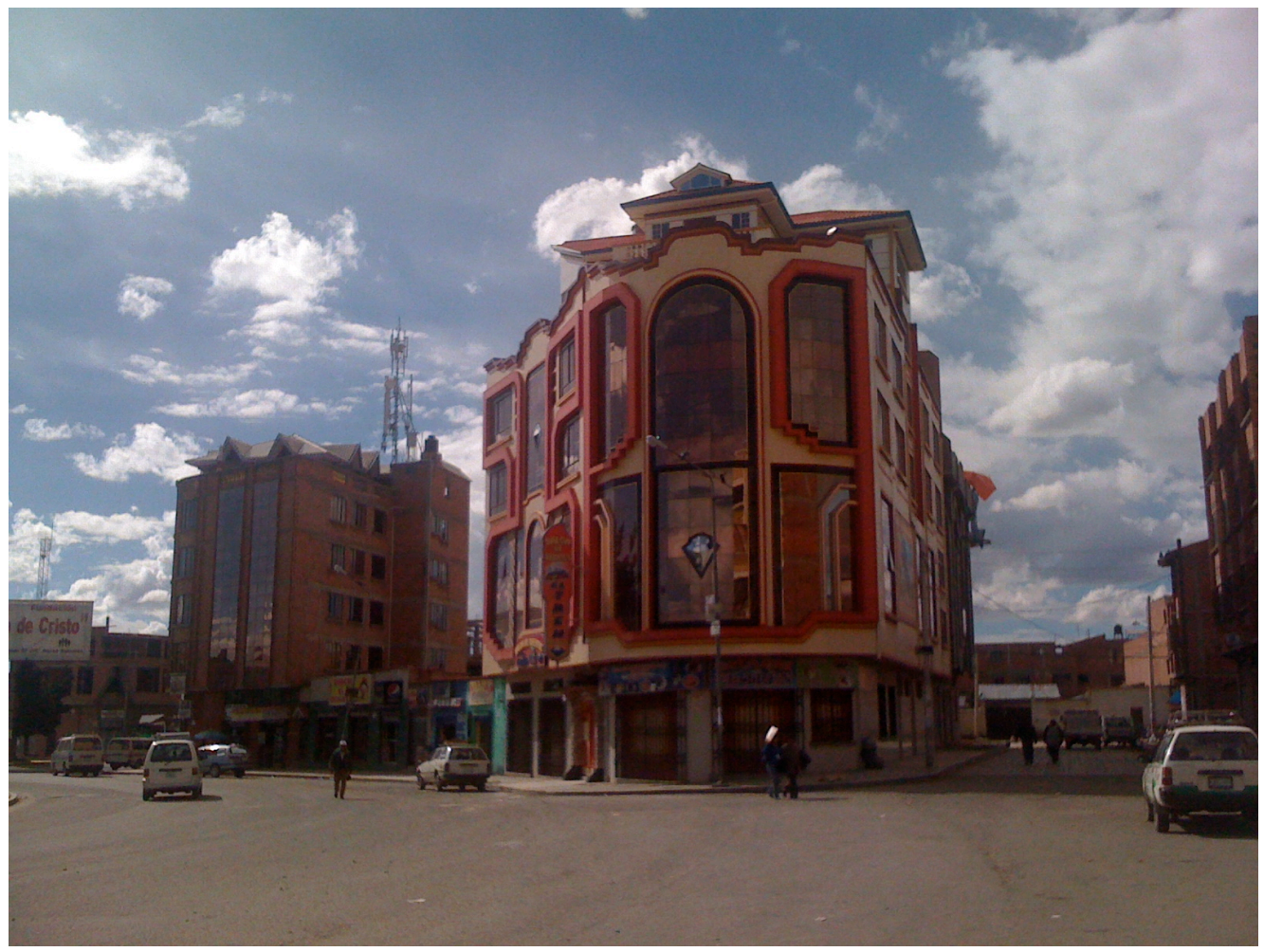

Ce sentiment d'incongruité provoqué par les chalets n'est pourtant pas spécifique au contexte de El Alto : Jean Bazin a ainsi décrit en quoi la présence d'un chalet basque dans une rue pavillonnaire de la périphérie parisienne remplit une fonction de message «mythique » qui exprime la «basquité » du bâtiment et de ses propriétaires : le sentiment immédiat de se trouver face à quelque chose qui «fait basque » alors qu'il se trouve «déplacé » (en termes de lieu et de sens) dans un contexte inédit, témoigne de l'irruption d'une étrangeté relative par rapport aux formes indigènes des autres pavillons alentours. Selon Bazin, «notre chalet basque dans sa rue de banlieue est donc un chalet basque détourné en exemple de basquité, (...) telle qu'elle se manifeste dans un certain style architectural et décoratif - style qui se présente comme une forme disponible offerte à la consommation sur un marché mondial du "style ethnique"" (Bazin, $2008:$ 153).

Dans ce cas, il s' agit moins de la transposition d'un habitat matériel que d'un modèle distinctif et d'une modification de signes, renvoyant à une «marque identitaire », un « signal-maison » qui compose une «figure, plus ou moins enrichie ou appauvrie, schématique ou élaborée, simplifiée ou complexe, une série de traits (boiseries brunes, façade clayonnée, etc.) identifiant comme relevant du style basque » (Bazin, 2008 : 154). Alors que le chalet dans un lieu incongru se contente de «faire basque », le pays basque existe comme «lieu originaire » de l'identité qui constituerait la « vérité première du chalet basque dont chaque signal architectural de basquité de par le monde serait le déplacement et l'altération "mythique" » (Bazin, 2008 : 156). Cette « couleur locale » serait ainsi un signal adressé au regard des autres : «il y a un côté désespérant dans toute identité ethnique, c'est qu'elle n'existe que pour les autres, et donc par d'autres, qu'on ne peut donc pas nettement distinguer être basque et faire basque, qu'être basque c'est toujours se conduire comme un Basque, faire sans cesse la démonstration de son être basque, adresser à la cantonade des signaux de basquité » (Bazin, 2008 : 161).

Les chalets de El Alto ne font pourtant ni basques, ni suisses : une fois passé le premier moment de surprise, ils s'imposent à la perception comme alteños, caractéristiques de El Alto. Alors qu'il semble de toute évidence faire référence à une culture « autre », le chalet semble pourtant instaurer, à El Alto, comme une présence déplacée ou, plus exactement, un détournement du sens d'une forme architecturale. Sa signification ne peut être assimilée à une «altération mythique » que si l'on occulte les usages sociaux dont cette architecture fait l'objet. Ici, ce n'est pas un chalet entre d'autres pavillons, c'est un chalet au sommet d'édifices ordinaires 
d'une ville andine, et qui donne à la résidence un aspect extraordinaire, la marque de son propriétaire, le signe de sa réussite économique et de son pouvoir social - de la même façon que certaines décorations architecturales révélaient, comme l'écrit Marcel Mauss dans son Manuel d'ethnographie (1926), « la présence d'une aristocratie ». On demandera au propriétaire l'explication de chaque décoration. Pareille enquête révèlera souvent, avec l'emploi de blasons, la présence d'une aristocratie. ». Les chalets sont des « architectures émergentes » à la fois locales, insérés dans leur contexte urbain, et globales, liés à des processus d'import-export de biens symboliques.

L'objectif de cet article est d'analyser la signification sociale de ces formes architecturales, dont l'implantation locale semble renvoyer à une dimension plus « globale » d'un point de vue culturel. Pour analyser la spécificité de ces constructions vernaculaires, qui sont des « architectures sans architectes » (Rudofsky, 1987), on reprendra tout d'abord différents cadres interprétatifs, centrés sur l'expression indigène des cultures locales ou au contraire sur l'internationalisation des formes culturelles. On explorera finalement l'hypothèse, formulée par Pierre Bourdieu (1967) à partir des travaux d'Erwin Panofsky (1951), d'une homologie entre les schèmes de pensée collectifs et les formes architecturales d'une époque, afin de déterminer les conditions sociales d'émergence des «chalets » dans la ville de El Alto. On cherchera ainsi quel type d'institution est susceptible de produire des schèmes communs au principe de ces formes indissociablement matérielles et symboliques.

\section{Une architecture « andine »?}

\section{La bourgeoisie chola : une économie ostentatoire}

L'intérêt des formes architecturales est, selon Marcel Mauss (1926), que « l'habitation caractérise une civilisation plutôt qu'un territoire déterminé. L'architecture apparaît ainsi comme l'art type, créateur par excellence ». Il ne faut pas pour autant en faire l'expression d'un groupe social ou d'une société : «L'enquêteur, précise Mauss, ne cherchera pas de prime abord la maison-type : chaque maison a son sens », et il faut au contraire étudier « toutes les variations individuelles et toutes les variations locales » en considérant leurs usages : « au terme de cette étude seulement, on pourra dégager la notion de maison-type sans risquer de confondre une maison de riche avec une maison de pauvre ». Les préceptes méthodologiques de Mauss mettent en garde contre l'assimilation des formes architecturales à l'expression d'une identité culturelle - en d'autres termes, contre le sentiment évoqué plus haut de considérer les chalets de El Alto comme des phénomènes «typiquement andins ». Ils invitent en revanche à rapporter les caractéristiques architecturales aux pratiques sociales dans lesquelles elles s'inscrivent ${ }^{2}$, et les « habitudes » dans lesquels elles s'inscrivent (Teyssot, 2013).

Les chalets de El Alto présentent une véritable « unité dans la diversité » : au-delà des différences de forme, de couleur, de matériaux, les habitations construites au sommet des immeubles renvoient à un même "style » architectural, et donc, peut-on supposer, à un même groupe social, capable d'en supporter les coûts économiques de fabrication. De plus, les édifices ont une grande visibilité due non seulement à leur taille, à leurs couleurs souvent éclatantes et contrastées, à leurs formes asymétriques et à l'effet de surprise qu'ils causent, mais aussi à leur emplacement prééminent sur les avenues très fréquentées et les carrefours situés principalement dans les quartiers du commerce et de l'artisanat de El Alto. Ces observations font écho aux remarques de Marcel Mauss, selon lequel « la maison n'a pas d'existence en soi normalement, sauf dans les pays où l'habitat est essentiellement dispersé, mais le cas est rare. L'étude de l'habitation ne serait pas complète sans une étude du village, ou de la ville, s'il y a lieu. Cette question, trop souvent étudiée en termes purement géographiques, se pose quant à moi au moins autant en termes statistiques et techniques» (Mauss, 1926). Une étude systématique de la localisation des chalets permet ainsi de préciser l'hypothèse déjà émise au sujet de l'appartenance des propriétaires à la catégorie des entrepreneurs du commerce, de l'artisanat ou des transports, qui seuls auraient les moyens financier de bâtir de tels édifices (Andreoli, D’Andrea, 2014 : 114-115). Ils sont en effet situés aux points névralgiques de l'activité économique de El Alto, aux principaux carrefours ou en surplomb 
de places de marché fréquentées : là où la dimension économique du bâtiment est pleinement exploitée, avec les ateliers ou les entrepôts de marchandise au rez-de-chaussée.

À El Alto, la réussite des entreprises familiales, dont plus de $90 \%$ comporte moins de quatre employés (CDILP, 2004), remonte aux politiques néolibérales appliquées en Bolivie à partir de 1985 (Gill, 2000 ; Kohl, 2004). Leurs activités relèvent moins de l'économie de marché que d'une «économie ethnique », au sens défini par Olivia Harris : une production de biens qui peuvent circuler hors marché à de larges échelles, et qui est basée sur l'organisation du groupe ethnique d'appartenance. La notion d'économie ethnique, qui a originellement été utilisée pour expliquer les économies rurales des communautés indigènes, peut être appliquée aux aires urbaines où les entreprises familiales continuent de structurer l'organisation des activités productives et commerciales. Ces populations indigènes ont été désignées, depuis le $\mathrm{XVI}^{\text {ème }}$ siècle, par le terme de «cholos » : issues des communautés aymara de l'altiplano bolivien, elles sont venues, depuis l'époque coloniale (Soruco, 2011), s'installer et travailler en ville, où elles ont constitué un groupe caractérisé par des métiers subalternes (emplois domestiques, commerce de rue, artisanat, etc.) dans les quartiers centraux mais par une implantation résidentielle circonscrite aux zones périphériques.

Les cholos sont généralement caractérisés comme un groupe social rejetant les valeurs dominantes de la bourgeoisie blanche et métis, notamment la modestie, l'esprit d'épargne et la modération: selon Nico Tassi, ils gèrent une «économie transnationale de consommation prospère et ostentatoire qui met l'accent sur les dépenses excessives, et qui matérialise le projet au plus vite dans des démonstrations d'abondance - qu'il s'agisse de vêtements, d'exhibition de biens ou de parades religieuses. En dépit de leur richesse économique, les cholos demeurent cependant un groupe discriminé par rapport au reste de la population blanche et métis des villes pour leur supposée incapacité à se soumettre aux lois de la rationalité économique » (Tassi, 2010 : 191). Si le principe d'abondance qui gouverne les pratiques économiques et religieuses des cholos renvoient à des valeurs cosmogoniques associées à la reproduction des biens possédés, c'est aussi ce principe qui leur procure néanmoins une entrée dans une forme d'économie de marché où l'excès, plutôt que la gestion de la rareté, apparaît constituer la motivation de l'échange.

Cette caractérisation de l'ethos cholo aide comprendre le paradoxe apparent des formes architecturales émergentes à El Alto. Occupant tout un niveau au sommet des immeubles, ils se caractérisent par un design architectural évoquant le chalet suisse, tout en présentant des caractéristiques éloignées de l'équilibre sobre et austère des chalets en bois des Alpes: formes anguleuses et pas toujours symétriques, couleurs vives et matériaux clinquants (marbre, métaux précieux comme des plaques de cuivre, etc.). Les ornementations esthétiques s'inscrivent dans une logique de démonstration du pouvoir, économique et social, des propriétaires qui mettent leur point d'honneur à personnifier leur édifice.

Les immeubles dans la totalité de leurs composantes ont en fait des fonctions économiques multiples, comme le montrent les photographies 2 et 3 prises sur l'avenue 16 de Julio, l'artère principale de El Alto menant au Lac Titicaca, avec le rez-de-chaussée utilisé pour le stockage des marchandises commercialisées par l'entreprise familiale ; une salle de réception qui occupe le premier voire les deux premiers étages, avec des baies vitrées larges et colorées ; des bureaux à louer au deuxième ou troisième étage et, au quatrième, des appartements, occupés par des membres de la famille ou des locataires; et enfin, au dernier étage, comme une sorte de penthouse, le chalet. Si l'organisation des étages inférieurs peut rappeler celle des édifices des riches membres des communautés de l'altiplano, le fait d'installer un espace de résidence au dernier étage marque une rupture avec les usages ruraux, où le toit reste un espace sacré (Arnold, 1992). 
Photographies 2. Avenue 16 de Julio

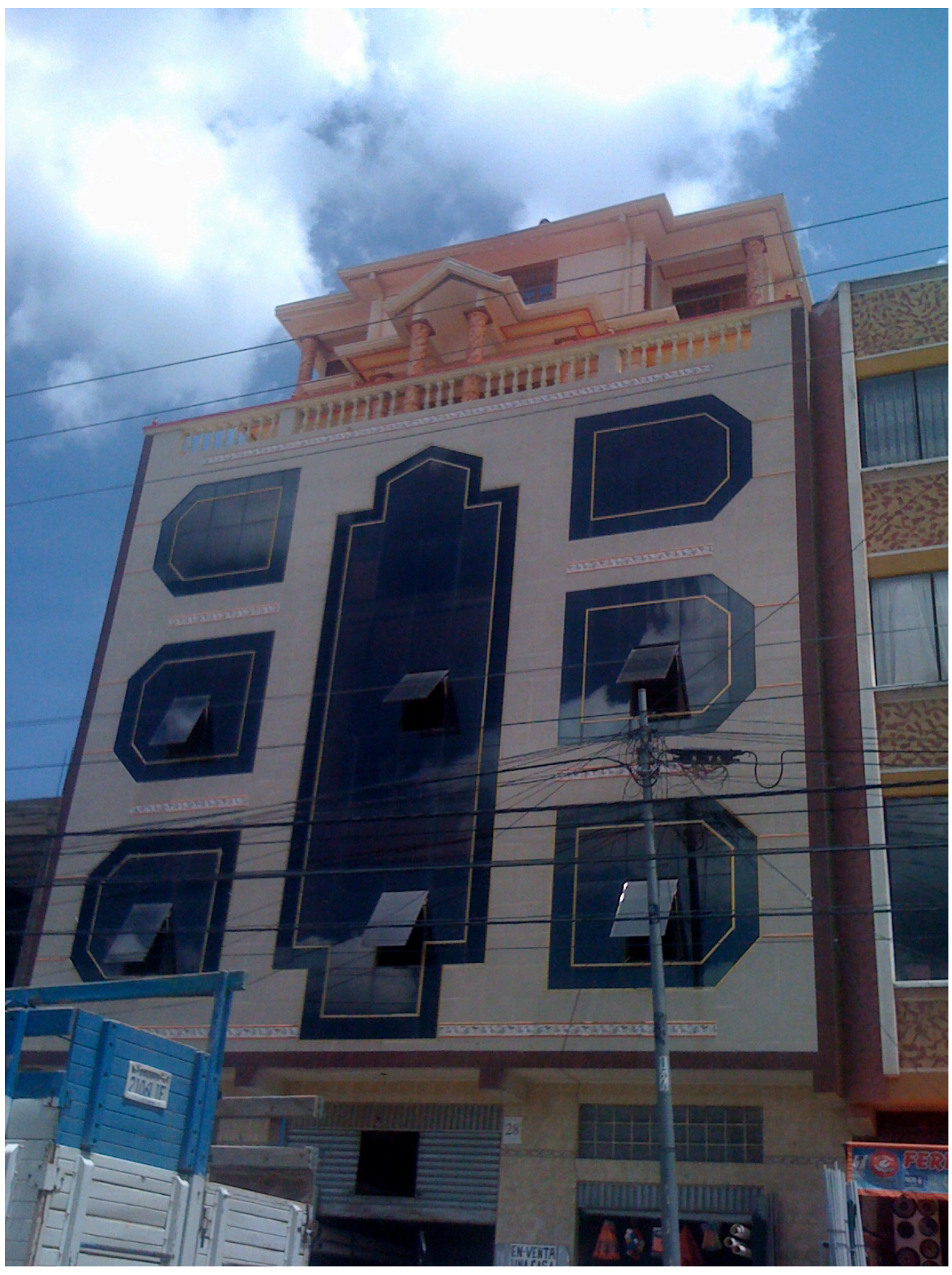




\section{Photographies 3. Avenue 16 de Julio}

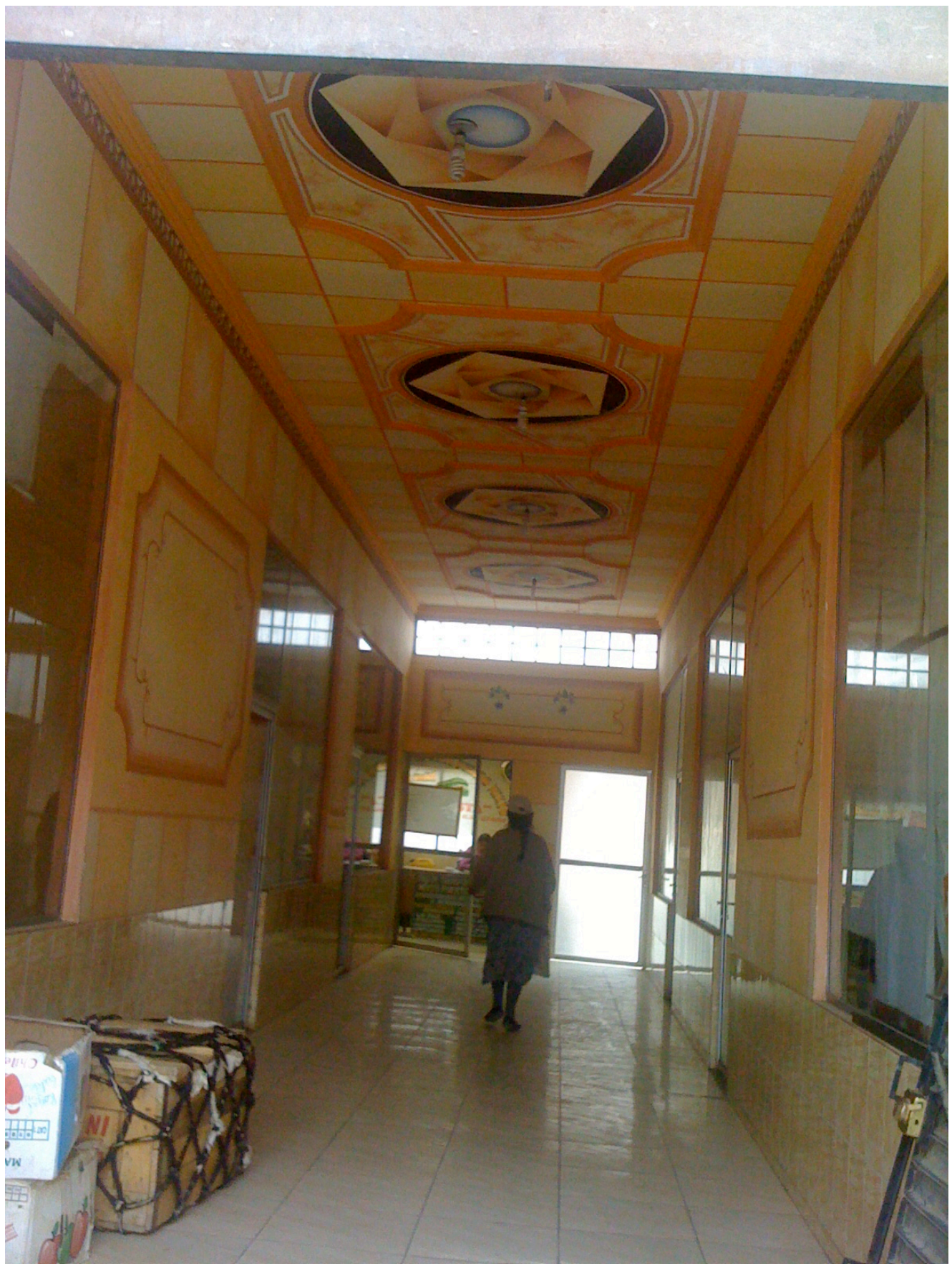

D'un point de vue symbolique, les chalets opèrent une dénégation de l'économie dite «capitaliste » telle que Max Weber l'a caractérisée : ils sont ostentatoires et représentent des dépenses somptuaires en regard de l'état d'inachèvement de la plupart des édifices alentours ou des infrastructures urbaines en contrebas. En même temps, ils se superposent à l'organisation économique du bâtiment. Ils sont faits pour être observés par les voisins afin de montrer la réussite individuelle de leur propriétaire, et de sa réussite supérieure celle des autres. Comme l'affirme un architecte de El Alto, « ce que les gens qui font construire un chalet veulent, c'est "comme mon voisin, mais en mieux..." ». Il serait pourtant réducteur de réduire la prolifération des chalets à un simple phénomène d'imitation, tant cette « unité dans la diversité » qui les caractérise semble renvoyer à un marquage de réussite individuelle. Dans quelle mesure cette volonté de distinction individuelle renvoie-t-elle alors à un groupe social déterminé ? Suffit-il d'invoquer ici l'identité culturelle d'un groupe dominant au sein des « sociétés andines »? 


\section{Les « identités ethniques » face au « rouleur compresseur de la modernité »}

La caractérisation des chalets comme un phénomène spécifiquement « andin » (Cárdenas, 2010; Neuman, 2013) consiste à en faire la manifestation par excellence de l'intense travail d'expression et d'invention culturelle qui se produirait dans la ville « indigène et rebelle » de El Alto. L'immeuble avec chalet en surplomb témoignerait de la persistance toujours vivace et créative des cultures aymara dans les espaces urbains qu'elles ont investies, de façon contrainte et subalterne, depuis la colonisation. Ces « architectures émergentes » témoigneraient des processus d'invention culturelle qui traversent cette ville «postcoloniale », fondée seulement au $\mathrm{XX}^{\circ}$ siècle, et à ce titre capable, plus que d'autres villes fondées par les Espagnols, de raviver une culture «précoloniale».

L'usage d'une catégorie comme «lo andino ( Harris, $2010: 10)$ présuppose une continuité culturelle entre les sociétés passées et présentes. On peut ainsi lire sous la plume de l'anthropologue bolivien Xavier Albó, que « le monde aymara, composante du monde andin, est un des peuples témoins qui a le mieux conservé sa vitalité. Malgré les processus de déstructuration et de déculturation dont il a souffert, comme les autres, son peuple et sa culture restent vivants » (Albó, 1988 : 22). Dans une même perspective, Robert Dover, introduisant un recueil de textes sur les cosmologies andines, affirme que les communautés locales gardent leur spécificité après cinq cents ans d'exposition forcée au système de pensée occidental. Cette préservation de l' « authenticité » serait en particulier facilitée par la capacité de la cosmologie andine à intégrer des éléments extérieurs : «Un discours cosmologique continu et persistant mène non à l'émergence d'un nouvel ordre social mais à un ordre social qui ne cesse de se manifester comme un phénomène spécifiquement andin. (...) Il y a une continuité structurelle dans la cosmologie andine à travers les éléments culturels nonandins deviennent systématiquement andins. L'authenticité est une interprétation possible du statu quo culturel. C'est aussi un objectif du discours cosmologique par lequel les éléments culturels non-andins deviennent des symboles manipulables dans la structure de la cosmologie andine $\gg$ (Dover, $1992: 2$ ).

Les communautés indigènes auraient donc la capacité de s'adapter continuellement au changement et à l'intrusion de cultures extérieures grâce à la persistance d'une cosmologie sans cesse réinterprétée et authentifiée comme spécifiquement « andine ». Cette interprétation culturaliste des chalets s'inscrit dans un contexte politique de réhabilitation des populations dites « originaires », à laquelle l'arrivée au pouvoir d'Evo Morales en 2005 a donné une dynamique institutionnelle et une légitimité politique. Les élites indigènes locales exprimeraient dans ces « inventions architecturales » leur «identité culturelle», au croisement de la «mémoire collective populaire » et de la modernité urbaine, assimilée à l'Occident capitaliste. Cette interprétation échoue cependant à saisir la spécificité de ces formes architecturales: une structure de bâtiment « traditionnelle » (plusieurs étages, caractéristiques de l'architecture adoptée par les familles en réussite économique de l'altiplano), qui incorpore des éléments «étrangers » (le chalet), pour créer une forme originale (le chalet sur le toit) et à contre-courant des critères de jugement esthétiques ordinaires - autant de facteurs qui font écran à l'analyse. La mise en évidence du caractère « andin » des architectures émergentes tend en effet à essentialiser une identité ethno-raciale qui, comme l'a montré Andreas Wimmer (2004), ne constitue qu'un principe secondaire de classification dès lors que les relations sociales dans lesquelles elles s'inscrivent sont ethniquement homogènes. Assimiler les chalets à l'expression d'une identité ethnique court donc le risque de naturaliser les traits distinctifs des cultures locales.

Il s'agit donc d'analyser le rôle que joue la référence aux «cultures andines » dans la production de formes culturelles nouvelles. Cette invocation de la «tradition » peut être interprétée comme un processus global d' « indigénisation de la modernité », au sens défini par Marshall Sahlins (2007), qui n'a pas parlé d'architecture mais dont le cadre interprétatif peutêtre transposé à ce type de biens. La réception de la marchandise occidentale ne déboucherait pas sur la destruction des cultures indigènes mais au contraire sur le développement des relations sociales traditionnelles et sur la réappropriation de l'échange marchand au travers des 
catégories propres à chaque cosmologie indigène. Les cultures traditionnelles subsisteraient sous l'hégémonie occidentale, et la capacité de résistance des peuples opprimés permettrait aujourd'hui de les réhabiliter. La continuité culturelle ne serait pas une sorte de résurgence du passé ou de pont jeté un peu miraculeusement entre les époques, mais le résultat du développement des rapports sociaux traditionnels par l'incorporation des éléments étrangers dans les schèmes culturels locaux. Le travail de Sahlins sur les îles du Pacifique consiste par exemple à montrer comment les biens occidentaux sont incorporés aux catégories des pouvoirs indigènes, qu'il s'agisse de marchandises européennes prenant la forme de signes de privilèges divins dans le cas des Kwakiutl, ou de l'exemple de l'interprétation du rôle de Charlie Savage et des mousquets dans l'expansion fidjienne de l'île de Bau : si « les mousquets furent "indigénisés" » (idem : 163), c'est parce qu'ils ruinaient la protection dont bénéficiaient les chefs dans les batailles et que leurs effets étaient amplifiés par les caractéristiques de l'ordre culturel fidjien, où le système hiérarchique faisait de la mort du chef un « désastre cosmique ». Bref, « ce ne sont pas les mousquets qui ont rendu les chefs fidjiens puissants, mais bien plutôt la chefferie qui a conféré leur puissance aux mousquets » (idem : 186). L'originalité de la théorie de l'indigénisation de la modernité est d'insister sur la dimension culturelle de ces processus d'adaptation :

«L'appropriation culturelle par les peuples de conditions extérieures qu'ils n'ont pas créées et auxquelles ils ne peuvent se soustraire est au principe même de leur action historique. Construit en relation avec les forces de la nature et les pressions exercées par les autres sociétés, chaque schème culturel historiquement avéré a été précisément le produit de cette contrainte pragmatique. Je ne prétends pas, évidemment, ignorer le rouleau compresseur de la modernité, je pense seulement que son déroulement historique doit être regardé comme un procès culturel. Le capitalisme occidental a déchaîné sur le monde des forces considérables de production, de coercition et de destruction. Pourtant, et précisément parce qu'on ne peut leur résister, les relations et les biens du système global prennent aussi des places signifiantes dans l'ordre local des choses. Lorsque cela se produit, les changements historiques de la société locale restent en continuité avec le schème culturel précédent, même si la nouvelle génération acquiert une cohérence culturelle d'un genre distinct » (Sahlins, 2007 : 206-207).

L'intérêt de la théorie de l'indigénisation de la modernité est de mettre l'accent sur la façon dont les sociétés locales s'approprient des éléments étrangers pour produire leurs propres formes culturelles : les « chalets » de El Alto, du fait de l'emprunt d'un symbole du capitalisme extérieur, permettent de créer une forme symbolique spécifique, caractéristique des cultures andines. Mais c'est cette prééminence accordée à la culture sur les processus sociaux qui constitue cependant la limite de la thèse de l'indigénisation de la modernité : l'appropriation des marchandises et des modes de vie occidentaux sous des formes « traditionnelles » ne peut se résumer, comme le remarque Alain Babadzan, à « la réaffirmation de principes cosmogoniques qui auraient traversé l'histoire en endossant des habits neufs » (Babadzan, 2009 : 111). Si Sahlins remet en cause, à juste titre, l'opposition entre coutume et rationalité, tradition et développement, en montrant que la culture est un processus et que la continuité peut s'opérer à travers le changement, il reste dans le cadre d'une vision de la continuité culturelle qui peut aussi bien relever d'un « conservatisme » prônant le retour aux traditions ensevelies sous la corruption de la globalisation capitaliste, que d'un «postcolonialisme radical » pour lequel l'hégémonie de la modernité ne parvient pas à domestiquer totalement l'autonomie des peuples indigènes. La théorie de l'indigénisation de la modernité pense les transformations économiques et sociales dans le cadre de schèmes culturels des populations colonisées: ou bien ces schèmes restent stables en incorporant les éléments extérieurs (résistance), ou bien ils se maintiennent en se modifiant (appropriation). Dans tous les cas, la culture reste un facteur déterminant en dernière instance.

Ce modèle explicatif culmine dans l'idée d'une « culture des cultures » traduisant une nouvelle organisation planétaire: « unifié par l'expansion du capitalisme occidental au cours des derniers siècles, le monde est aujourd'hui re-diversifié par les adaptations indigènes au rouleau compresseur de la globalisation. Dans une certaine mesure, «l'homogénéité globale et la différenciation locale se sont développées ensemble, la seconde répondant à la première au 
nom de l'autonomie culturelle indigène », ce qui définit "un système mondial composé de divers modes de vie » (Sahlins, 2007 : 317). La résistance des sociétés non occidentales à la globalisation et à l'impérialisme opèrerait un détournement de la signification culturelle des marchandises, qui ne serait pas incompatible avec la coexistence du capitalisme au sein des sociétés traditionnelles. L'assimilation des formes architecturales à une culture locale relève finalement d'un réductionnisme culturel qui tend à occulter la domination exercée par les rapports sociaux du « capitalisme global ». La résistance des peuples indigènes devient alors pour le moins difficilement explicable, sauf à adopter le postulat psychologique d'une «humiliation» de ces peuples qui se retourneraient vers la dimension la plus spirituelle de leurs cultures « originaires », cultures qui auraient résisté au matérialisme moderne : « Postuler a priori que la cosmologie est encore et toujours au poste de pilotage, qu'elle détermine l'orientation du rapport aux marchandises et par là à la modernité, empêche de donner toute leur place à des déterminations d'ordre sociologique » (Babadzan, 2009: 111). Elle empêche, dans le cas des chalets, de prendre la mesure des rapports sociaux qui font des élites cholas de El Alto un groupe dominant localement.

\section{La circulation internationale des formes architecturales}

\section{Hégémonies architecturales}

S'il faut saisir les innovations locales non seulement comme des processus culturels mais aussi comme des rapports sociaux de pouvoir, il s'agit alors de prendre en compte la dimension symbolique de la domination, comme l'ont fait Reinhard Bentmann et Michael Müller (1970) au sujet de l'émergence de la villa italienne du XVIème siècle, dans le contexte de la croissance économique de Venise, en reprenant un cadre d'analyse gramscien en termes d'hégémonie. Si leur travail porte sur des formes élaborées de construction réalisées par des architectes érudits qui n'ont pas d'équivalent dans la fabrication des « chalets » de El Alto, l'intérêt leur objet d'étude par rapport au cas bolivien est de montrer dans quelle mesure la référence au monde rural peut fonctionner comme une légitimation du pouvoir des élites locales.

La villa de la Renaissance italienne incarne, selon Bentmann et Müller, une combinaison des forces économiques, politiques et esthétiques de son époque : elle constituerait une « transfiguration idéologique » par laquelle un groupe de propriétaires terriens de la bourgeoisie urbaine aurait défendu son contrôle sur les latifundia nouvellement constitués dans la campagne alentours. L'émergence de la villa n'est donc pas seulement rapportée à la logique économique du capitalisme vénitien naissant, mais à un groupe social particulier (la bourgeoisie émergente du capitalisme italien) dans le contexte plus large d'un conflit entre la ville et la campagne : bien plus qu'une invention architecturale, elle incarne à la fois une idéologie et une «utopie négative » pour les plus fortunés. Dans un contexte de concentration urbaine croissante, une classe sociale en ascension utilise le paysage de la campagne comme une décoration pour la villa, et de façon plus générale reprend les symboles de la domination de l'ancienne noblesse féodale : elle utilise les formes classiques de l'art antique pour réaliser une sorte de "paradis sécularisé sur terre », contre les conflits associés à la vie urbaine : «l'architecture se situe à l'intersection du cosmologique, de l'astrologique et des perspectives sociales » (Bentmann \& Müller, 1970 : 92). Si la villa reproduisait, sur le plan architectural, les structures de la domination dans la Venise de la Renaissance, cette actualisation de formes esthétiques du passé renvoie aussi au « rêve de la campagne » comme idéologie atemporelle et comme posture conservatrice : dans l'Angleterre des $\mathrm{XVIII}^{\circ}$ et $\mathrm{XIX}^{\circ}$ siècles par exemple, en pleine phase d'industrialisation, les propriétaires de fabriques transférèrent leur lieu de résidence à la proximité immédiate des usines de production : ils actualisèrent donc la forme de la villa pour exprimer leur pouvoir économique et social.

La caractérisation de l'« architecture hégémonique » aide à comprendre la complexité des relations, à la fois coercitives et idéologiques, qui constituent l'esthétique architecturale comme un indicateur des transformations du pouvoir. La villa comme architecture hégémonique révèle ainsi « l'assurance de soi de ceux qui utilisent encore les pinacles et les tours comme des symboles de leurs revendications sociales et politiques » (idem : 106). Ils font 
ainsi l'hypothèse que le penthouse (derniers étages), comme les villas, recèle une « hostilité face à l'urbanisation » (idem : ch.19): ils expriment un « rêve de fuite hors de la ville » et un « désir non dissimulé de symboles de statut » :

«The penthouse is situated high above the netherworld of common existence and on top of the
flat roof of the tower block. As such, it satisfies the demands both for a luogo privilegiado and
for a sito elevato, which where postulated by Renaissance theoreticians with regard to the villa
as hegemonic architecture. (...) Besides the function of surveying and overseeing, which the
penthouse shares with the villa owing to a sito elevato, there is also the idea in common of a
prospect, or belvedere. [...] In its contemporary form, the penthouse too is antiurban, as long as
it remains a privileged site for a few wealthy individuals » (idem : 135-136).

Toutefois, le cadre d'analyse gramscien des auteurs réduit le contexte social à un déterminisme économique dans le cadre duquel une nouvelle élite utilise une idéologie anti-urbaine et l'architecture qui lui correspond pour exprimer sa domination. Ce schéma d'interprétation, s'il permet de comprendre les rapports de domination dans lesquels s'inscrit la référence au monde rural, est au final difficilement transposable aux chalets de El Alto, pour plusieurs raisons. Tout d'abord, la revendication d'appartenance à une communauté rurale de l'altiplano, si elle peut-être assimilée à une forme de "rêve de la campagne », n'efface pas le désir de rester inséré dans les relations sociales de l'économie urbaine : les chalets, qui reprennent un symbole de prospérité économique occidentale, sont construits au sommet des édifices familiaux, et non dans une propriété champêtre à l'écart. D'autre part, ce cadre d'analyse ne permet pas de comprendre l'appropriation d'éléments étrangers au sein des rapports de pouvoir locaux. Alors que la théorie de «l'indigénisation de la modernité » les dissout dans les cultures locales, et que la théorie de «l'hégémonie architecturale » en ignore largement l'importance, ce sont donc les modes de circulation internationale des formes architecturales qu'il faut explorer.

\section{«Culture globale » de l'architecture et domination coloniale}

L'idée d'une global culture en architecture repose sur une analyse de la contribution de la matérialité spatiale des bâtiments à la formation des identités sociales. Selon Anthony King (2004 : xiii), « a particular type of individual and consumer-oriented form of outer suburbanization, as well as occasional leisure space, represented by a distinctive (though varying) form of one storey dwelling (everywhere referred to the same term, "bungalow"), had developed in 'advanced' and especially postindustrial societies ». Ainsi, dans son étude sur la diffusion internationale du bungalow, de l'Inde à la côte ouest étasunienne, King (1984) caractérise-t-il les rapports de force coloniaux qui structurent la circulation des formes architecturales ; le bungalow y est défini comme «the one perfect house for all tropical countries ». Cette architecture tropicale a ainsi fonctionné comme un marqueur d'identités nationales lors de son importation à travers les espaces globaux de la métropole et de ses « postcolonies ». De même, Abbidin Kusno mentionne, à partir de son étude de l'espace urbain en Indonésie, que

« to achieve translocal regionalist architecture, the preceding forms of dominance represented by modernist architecture will thus have to be rearticulated (...) In a self-inscribed alterity to the modernist-international architecture, the category of 'tropical' climate becomes a symbol for a locally-meaningful architecture. (...) This represents a process of architectural 'localization,' a cultural negotiation of the authority of the international style» (Kusno, $2000: 200$ ).

L'impact de la domination coloniale sur la production de l'espace (Blais, 2009) révèle ainsi un processus d'homogénéisation croissante de l'architecture mondiale, matérialisée au XXème siècle, d'abord par les maisons individuelles puis par les gratte-ciels, dotés d'une fonction symbolique évidente d'imposition de «grandeur » nationale. Dans ce cadre d'analyse, les développements architecturaux viennent toujours de l'extérieur : il y aurait en fait une corrélation entre les représentations concurrentes, au niveau mondial, de l'architecturespectacle, et la hiérarchie des États-nation : « the shaping of the built environment is a writing of the history of a nation » (Kusno, $2000: 201)$. L'architecture comme vecteur de la global culture est ainsi intimement associée à la domination capitaliste, qu'elle fasse l'objet d'une acceptation tacite ou d'une résistance explicite. Dans cette perspective, les chalets peuvent 
apparaître comme le produit de l'importation d'esthétiques étrangères, dans un contexte de globalisation de l'économie, de la culture et de l'architecture. Mais ils n'expriment pas tant une résistance de la culture indigène qu'une conversion des élites locales aux logiques de capitalisme transnational et de ses symboles de succès : le chalet représentant la prospérité du « pays banquier» de l'Europe, la Suisse. Coïncidence qui n'est pas si anodine : le restaurant le plus « huppé » des quartiers prospères de La Paz s'appelle «La Suisse », et son blason est un chalet, comme le montre la photographie ${ }^{\circ} 4$. Cette vision de l'architecture des chalets comme appropriation d'un symbole de prospérité occidentale repose sur un modèle quelque peu mécanique de que « le capital symbolique n'est pas tant créé que transféré d'un espace de référence à un autre » (Dovey, 1999 : 121).

Photographie 4 : Représentation d'un chalet dans le restaurant « La Suisse » à La Paz. Selon Marcel Mauss, le détail des décorations, et l'usage de blasons, révèlent souvent « la présence d'une aristocratie. »

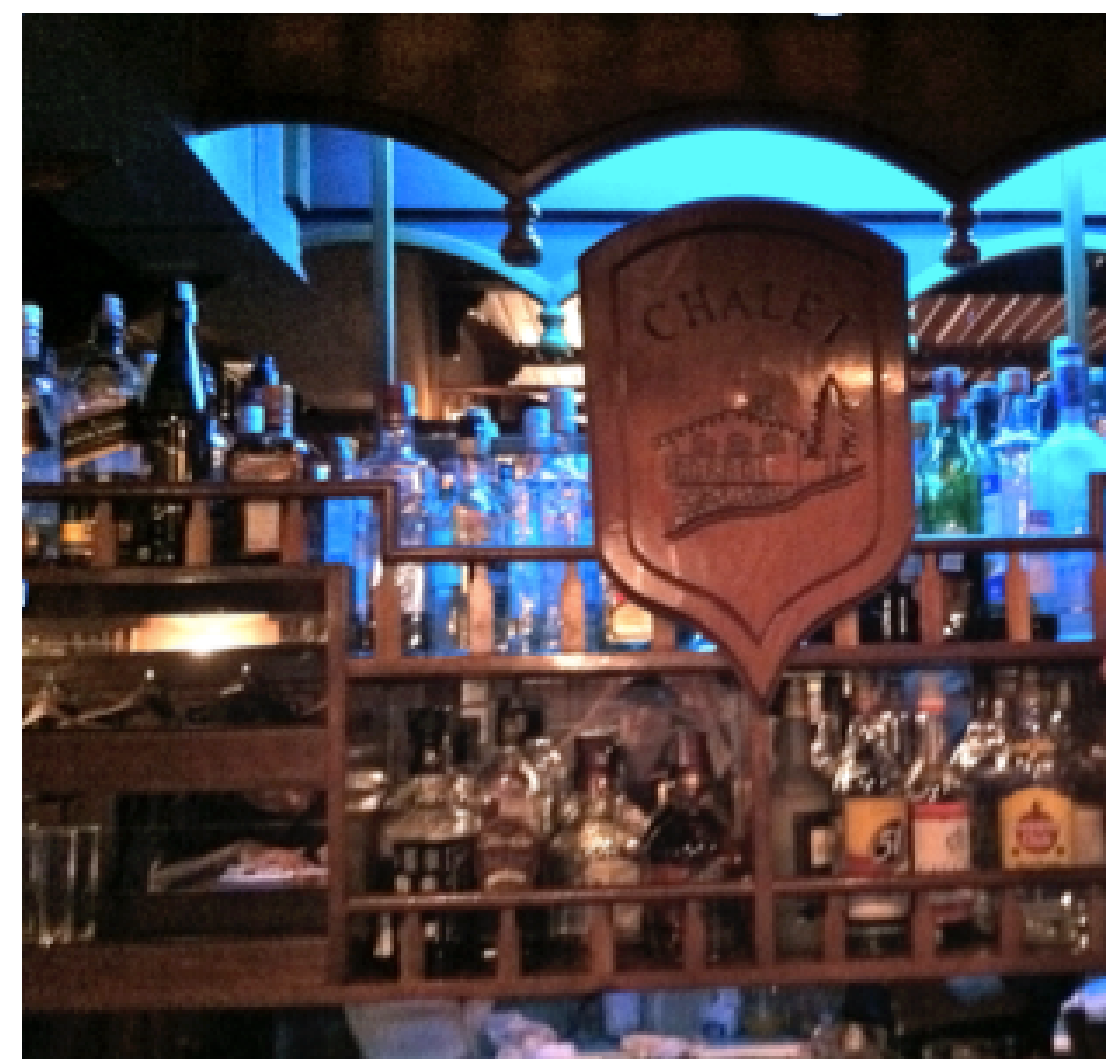

Si les approches postcoloniales de l'architecture globale permettent de comprendre le rôle de l'espace et de l'environnement urbain dans la reproduction des rapports de domination, elles s'attachent peu à la spécificité des pratiques locales de construction et d'ornementation des bâtiments: elles laissent en fait peu de place aux processus d'appropriation ou de détournement des significations des formes architecturales importées. Finalement, le cadre interprétatif de la global culture ne rend pas compte de la complexité des processus d'importation (ou inversement d'exportation) des biens symboliques : les logiques locales d'appropriation ne peuvent être assimilées à la circulation de formes globales qui resteraient uniformes dans chaque contexte, et il faut restituer la part de glissements de sens, sinon de création, révélée par cette appropriation. Même une approche intégrant les conflits de pouvoir au sein des populations locales, comme l'a fait Brenda Yeoh dans sa recherche sur l'organisation urbaine de Singapour depuis le XIXème siècle, peine à saisir de tels désajustements. Yeoh tente en effet de comprendre les « récits » de la domination et de la résistance des groupes indigènes : «The urban built environment refers to what David Harvey defines as the totality of physical structures-houses, roads, factories, offices, sewage systems, parks, cultural institutions, educational facilities and so on. Colonial landscape ideally reflected the power and prestige of the colonialists (...) and it is structured to enhance the flow of economic activities such as trade and communications which were crucial to the entire colonial economy» (Yeoh, $2003: 16$ ). 
De même, les fortes résistances des résidents contre l'installation de vérandas dans les rues de Singapour à l'ère coloniale révèlent l'impact des forces économiques sur la structuration des espaces publics et sur l'élaboration de nouvelles formes architecturales (Myers, 2003). Mais là encore, ces conflits ramènent la circulation des formes architecturales à des mécanismes d'importation homogènes, au sein de rapports de domination non moins uniformes.

\section{Les principes générateurs des pratiques architecturales}

\section{L'appropriation de formes architecturales étrangères}

L'analyse de la circulation internationale des formes architecturales doit inclure plusieurs facteurs, qui ne sont présentés que séparément dans les cadres interprétatifs présentés jusqu'à présent : les rapports de domination propres à leur contexte d'importation, mais aussi les vecteurs symboliques et esthétiques de leur réception. Ainsi Heliana Salgueiro montre-t-elle, dans le cas de Belo Horizonte au tournant des XIXème et XXème siècles, comment des formes architecturales «progressistes », empreintes d'idéologie hygiéniste, se sont imposées contre le baroque des constructions portugaises et l'anarchie des petites demeures. L'usage d'éléments inspirés des chalets européens est alors considérée comme «inadapté au pays » et en «dysharmonie avec le climat» (Salgueiro, 2007 : 275). La diffusion de la forme chalet dans le Brésil de l'époque se fait alors par le biais de ses éléments décoratifs les plus saillants : «Dans les maisons traditionnelles, on modifie l'inclinaison des toitures ou l'on ajoute des petites pavillons ou belvédères dont les bordures des toits sont ornementées de lambrequins » (idem : 275 ; l'auteure s'appuie ici sur Daly, 1972). Il subsiste donc un décalage consciemment entretenu, voire critiqué par les observateurs, dont Heliana Salgueiro rapporte les commentaires : l'un, affirmant que «nous n'avons pas de chalets, mais des maisons avec des becs ", l'autre rappelant que «le chalet n'est pas un style mais un type, une variante d'origine exclusivement suisse » qui a été « adaptée aux formes pittoresques comme maisons de riches pour les régions du nord de l'Europe ». Lorsque cette typologie architecturale se répand au Brésil, elle lie alors le cottage anglais à la référence suisse, comme autant de principes d'importation de « fragments » qui servent à « enjoliver les maisons urbaines comme celles des rues commerçantes de Rio [...]. La version brésilienne du chalet correspond donc à un cas de juxtaposition éclectique, dans la mesure où l'on assimile les innovations techniques industrielles arrivées sous forme d'éléments décoratifs, notamment les lambrequins en zinc, bois ou fer forgé et les tympans stuqués inscrits sur le pignon des façades avec des motifs floraux Renaissance » (idem : 225).

Avant de disparaître des espaces urbains dans les années 1910, cet éclectisme se diffuse comme une forme de transgression de l'opposition entre le «classique » et le «pittoresque », le « moderne » (occidental) et la « couleur locale ». Par comparaison, les chalets de El Alto semblent avoir une signification sociale quelque peu différente, qu'il s'agit maintenant d'analyser: moins tournés vers l'ajout d'accessoires décoratifs que vers l'édification ostentatoire d'un étage supplémentaire et en surplomb, ils s'inscrivent dans l'économie symbolique d'un bâtiment qui est subordonnée aux fins d'une entreprise familiale à la fois très implantée dans les réseaux de l'informalité et très connectée aux flux de biens internationaux. Dans cette perspective, ce n'est sans doute pas une coïncidence si les entrepreneurs de confection de El Alto ont développé, depuis quelques décennies, une forte relation avec le Brésil, où une forte migration de travailleurs boliviens est utilisée dans les fabriques de Sao Paulo (Vidal, 2012). Jean-Pierre Garnier et Roselyne de Villanova, remarquent ainsi que « loin de s'en tenir au classique effet d'imposition directe exercé par le colonisateur sur le colonisé, les riches commerçants et les membres des castes aisées ont souvent joué le rôle de "passeurs" de modes architecturales » : effet indirect de la migration et de la réussite des propriétaires boliviens des fabriques de textile, les chalets s'inscriraient au sein d'une « architecture de l'entre-deux », et qui est « disqualifié comme producteur de mélanges, qualifié de bâtard, de style impur et, en architecture, d'absence de style » (Garnier \& Villanova, 2003, 171-172). 


\section{À la recherche d'homologies : architecture chola et fraternités andines}

Afin d'éviter les réductionnismes culturel ou économique précédemment évoqués, on peut replacer les formes architecturales dans les pratiques sociales des groupes qui les promeuvent, conformément à l'intuition de Mauss (1926). L'approche iconologique d'Ervin Panosfky (1967) semble ici la plus heuristique. Elle a en effet pour but de trouver des homologies entre les structures des systèmes symboliques d'une société et les principes de conversion formelle entre ces systèmes. L'important est alors d'établir une « connexion concrète » qui rende raison de la logique de ces homologies, c'est-à-dire, pour reprendre les termes de Pierre Bourdieu, un habitus «par lequel le créateur participe à sa communauté et à son temps, et qui guide et dirige, de façon méconnue de lui, ses actes de création apparemment uniques » (Bourdieu, 1967 : 157). Dans le cas étudié par Panofsky, « les principes qui définissent les processus de la pensée scolastique comme les formes de l'espace architectural » produisent « des œuvres qui sont à la fois originales et réductibles à des schèmes plus généraux » (idem). L'application des principes d'analyse de Panofsky aux chalets andins ne va pas de soi, dans la mesure où il s'agit, comme pour les villas de la Renaissance précédemment citées, de formes culturelles érudites qui ont en apparence peu en commun avec la dimension vernaculaire des architectures andines. Cependant, dans la mesure où le modus operandi révélé par l'organisation matérielle des chalets relève de processus qui n'appartiennent pas aux seules logiques professionnelles de l'architecture, il est possible de rechercher des «formes concrètes » propres aux mondes andins, en s'interrogeant sur le type d'institutions qui serait structuralement équivalent à la scholastique.

Les entretiens menés avec les architectes révèlent le rôle décisif du propriétaire et du chef de travaux (maestro albañil) dans la conception et la mise en œuvre de chaque édifice. De fait, les architectes ne sont employés que pour des tâches techniques (calculs, matériaux) et juridiques (légalisation de la construction auprès des autorités municipales). Une forme de mépris professionnel émerge donc chez les architectes travaillant à El Alto, à propos des propriétaires qui ne connaissent pas les règles minimales de la cohérence architecturale : « les propriétaires veulent un "collage" de tout ce qu'ils voient et qui leur plaît, afin de le mettre dans leur maison... c'est la même chose avec les couleurs, il n'y a pas de critères » (Architecte, El Alto). Ce manque d'harmonie est donc considéré moins comme une expression de singularité et d'originalité du goût que comme un manque de compétence de la part de non professionnels - les chefs de travaux étant des artisans au service des propriétaires qui ont les moyens de les payer ${ }^{3}$. Si l'on considère les chalets comme l'expression de la réussite économique des entrepreneurs cholos de El Alto, il faut alors voir dans quelle mesure on peut dire que ceux-ci manifestent dans cette « visibilisation » de leur succès une forme de dénégation de l'économie caractéristique des élites cholas.

Le fait social le plus proche des chalets en matière d'ostentation des richesses n'est pas architectural : il est constitué par les processions et les défilés à l'occasion desquels ces élites cholas font de similaires démonstrations de réussite économique et sociale - rituels comme le Gran Poder, fêtes de quartier, célébrations universitaires, etc. Et si, comme le remarque Robert Darnton, une procession « ne peut être considérée comme la représentation fidèle d'une société car elle exagère certains éléments et en néglige d'autres », elle reste «l'expression de l'essence même de la société, de ses "qualités" et "dignités" les plus importantes »(Darnton, 2011 : 168). Ces événements publics, qui ont lieu à même la rue et bloquent régulièrement la circulation automobile, donnent lieu à un véritable étalage de ressources économiques : fabrication de costumes, location de fanfares avec de nombreux musiciens, mise à disposition de nourriture et surtout de boissons alcoolisées pour les participants, les invités et la famille, etc. Les groupes subventionnés par les prestes (les bienfaiteurs) doivent mieux figurer que tous les autres : non seulement en matière de danses ou d'animation mais aussi de luxe ostentatoire (Albó \& Preiswerk, 1991 ; Guss, 2006).

Les riches entrepreneurs cholos de El Alto sont parmi les plus importants financeurs de telles processions, pour la simple raison qu'ils en ont les moyens et qu'ils se doivent de «tenir leur rang » dans leurs communautés de référence, qui peuvent être leur communauté de quartier ou leur groupe professionnel. Si l'on veut donc trouver un équivalent andin de la vision du 
monde scolastique pour comprendre les principes de l'architecture chola, ce n'est pas du côté de l'institution scolaire qu'il faut chercher, car en Bolivie les élites indigènes n'y sont pas vraiment socialisées (Abercrombie, 1998 ; Widmarck, 2003 ; Martinez, 2010), ou plus précisément leur existence en tant que groupe social ne provient pas de la sélection scolaire. Il faut rechercher du côté d'un autre type d'institutions qui donne sens à leur pouvoir économique et social: les organisations de métier (syndicats, corporations) et de voisinage (comités de quartier), qui constituent des fraternités au sens défini par Max Weber (1982), et qui sont les véritables lieux de socialisation des entrepreneurs de l'élite chola.

Dans un autre contexte socio-historique, Max Weber a montré que de telles fraternités basées sur une activité économique se caractérisent surtout par leur façon d'occuper l'espace public. Si l'hypothèse de l'homologie entre chalets et processions s'avère justifiée, on peut alors affirmer qu'avec ces formes architecturales, les élites de l'économie ethnique localisées à El Alto concurrencent non seulement les administrations étatiques ou municipales, mais aussi et surtout leurs rivaux économiques pour l'occupation de l'espace public : mais ce faisant, elles déplacent la concurrence économique sur le terrain de l'ostentation des richesses. Dans cette perspective, elles participent d'un habitus économique dont la particularité est de dénier l'économie, au sens où elles sont des modes de consommation ostentatoires peu compatibles avec le protestantisme ascétique qui, selon Max Weber, a généré l'éthique capitaliste : ainsi les corporations médiévales étaient moins coercitives que les sectes protestantes, mais elles ne pouvaient tolérer l'investissement individuel dans la réussite économique valorisée par les sectes sous peine de détruire le principe même de leur cohésion sociale ; il fallait donc que les richesses soient redistribuées, sous une forme ou une autre, à la communauté, et les manifestations publiques (processions, fêtes, célébrations, etc.) en constituaient un moyen essentiel (Weber, 1906). Par conséquent, le succès individuel devait être dénié dans sa dimension économique pour permettre la conciliation avec les normes collectives.

Le principe d'abondance des fraternités cholas relève de la même ambivalence envers l'économie capitaliste ${ }^{4}$. C'est donc l'hypothèse d'un habitus économique déniant l'économie, propre aux fraternités (professionnelles ou résidentielles) des élites cholas, que l'on peut formuler pour expliquer la « connexion concrète » entre les principes des « architectures émergentes » à El Alto et les modes d'investissement de l'espace public de ces élites. Même s'il n'est pas possible de détailler l'analyse de ces constructions vernaculaires de façon aussi précise que l'homologie mise en évidence par Panofsky, par exemple, entre les croisées d'ogive et les césures de l'écriture gothique, il n'en reste pas moins possible d'identifier quelques caractéristiques concernant les mélanges de couleurs et de matériaux, ou encore la hiérarchie des formes : souvent, les chalets adoptent la couleur ou les alliances de couleur de la fraternité d'appartenance du propriétaire (Cárdenas, 2010); les formes des toitures et des ornementations, sont aussi tout en angles, en brisures, en surenchère de brillance. L'habitus étant un principe explicatif et générateur de la diversité des pratiques, il se retrouverait ainsi au principe de ses manifestations matérielles, qu'il s'agisse d'architecture ou de danse, dont les photos 6-7 donnent des illustrations. 
Photo 6 : défilé d'une fraternité de El Alto au Gran Poder à La Paz

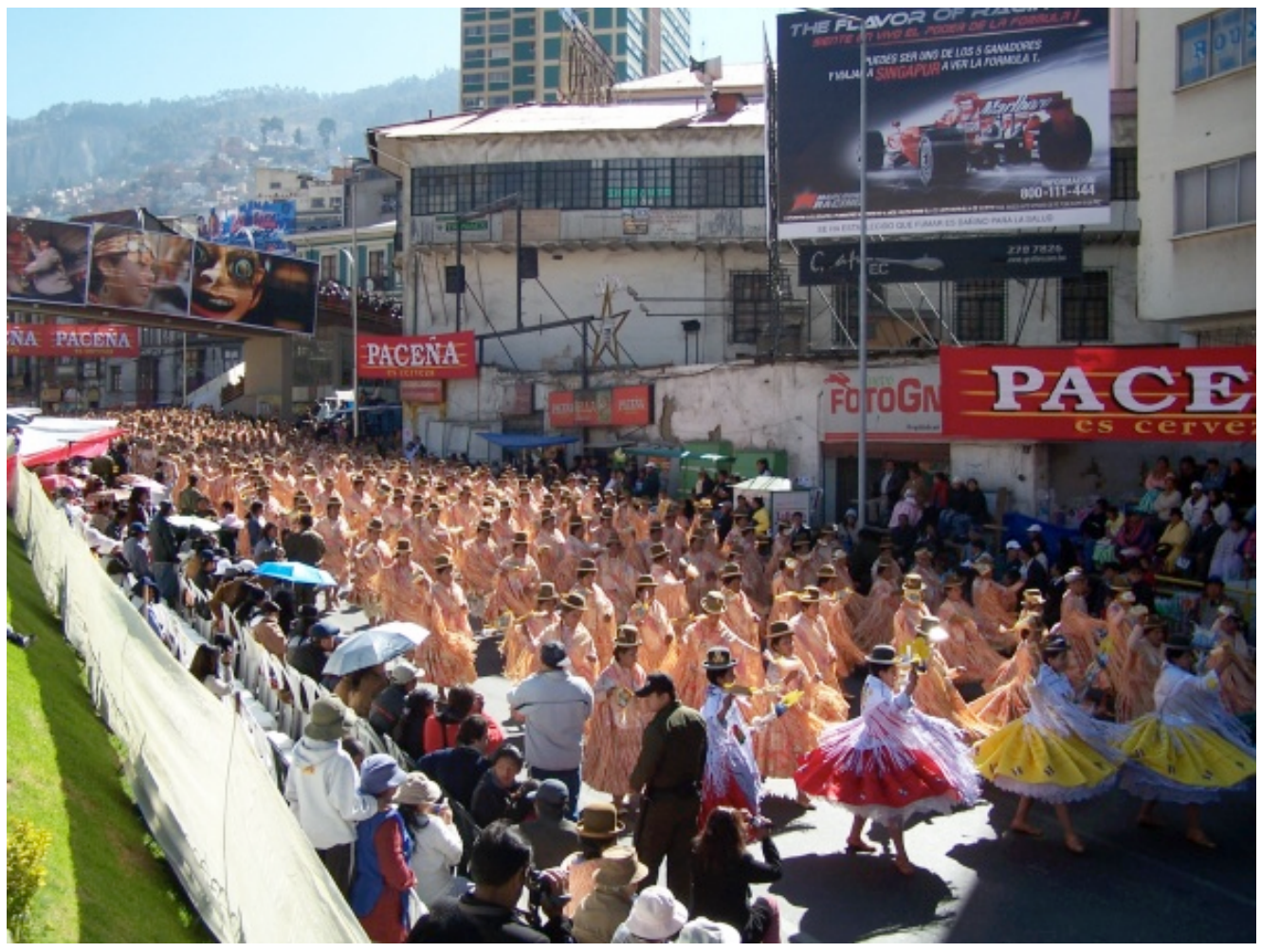

Photo 7 : défilé à El Alto

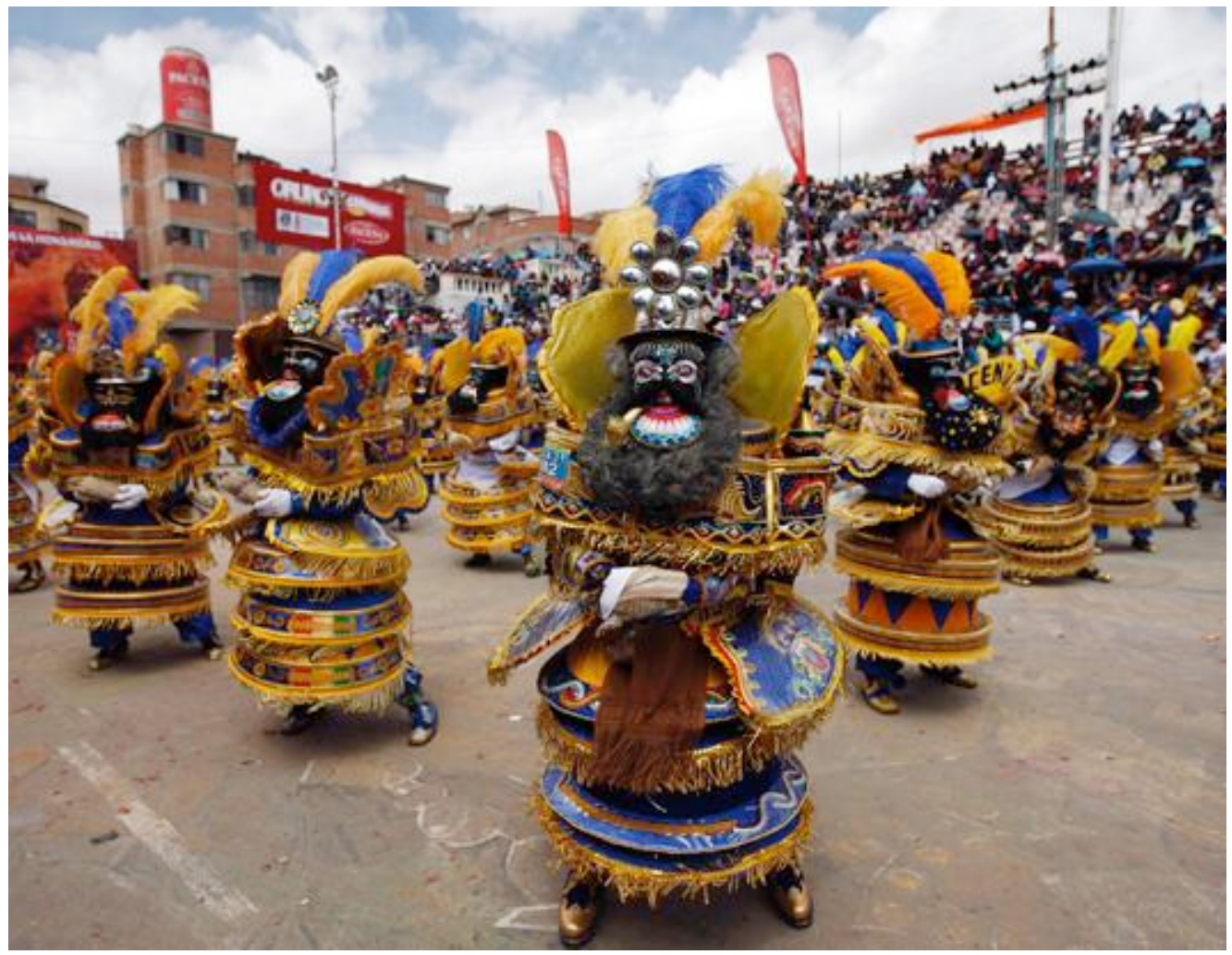

\section{Conclusions : un programme de recherche}

L'enquête exposée sur les chalets à El Alto consiste encore plus à formuler des hypothèses et des interprétations que des explications aisément testables et réfutables. Elle s'appuie cependant sur un cadre interprétatif qui rend compte à la fois de l'internationalisation des formes architecturales (étudiée par les théories de la global culture) et de la spécificité de leurs appropriations locales (sur lesquelles insistent les théories de l'indigénisation de la modernité), 
avec la mise en évidence d'un habitus que l'on pourrait qualifier d'ostentatoire, orienté vers la démonstration publique de richesses accumulées dans la sphère privée de l'économie familiale : un habitus au principe aussi bien des dispositions économiques que des pratiques festives et des choix esthétiques en termes d'ornementation. Elle rend compte de la connexion concrète entre l'émergence de constructions architecturales spécifiques et celle d'une élite économique qui ne cherche pas à trouver sa réussite dans la « certitude subjective de son élection divine », comme le notait Weber à propos des protestants à l'origine du capitalisme, mais qui cherche à exprimer son nouveau pouvoir social dans les cadres ostentatoires de son groupe de pairs, en insérant des symboles extérieurs prestigieux dans les formes culturelles vernaculaires des sociétés andines - et en leur donnant de fait une nouvelle signification sociale.

Plusieurs pistes de recherche se dégagent donc de cette hypothèse. Si le principe explicatif des formes architecturales réside dans les fraternités comme modes d'organisation économique, il faut donc étudier ce qui dans leurs principes pratiques d'existence collective (relations hiérarchiques, classifications comptables, schèmes de pensée et cosmologies implicites, etc.) se retrouve mis en œuvre dans l'esthétique des bâtiments. Autant d'éléments susceptibles d'alimenter les discussions sur les architectures vernaculaires (Rudofsky, 1987 ; Brunskill, 1006), dont les chalets bouleversent quelque peu l'appréhension, dans leurs dimensions à la fois globale et locale. L'enquête doit alors se centrer non sur les architectes, qui restent relativement extérieurs à la conception de bâtiments dont ils désapprouvent l'esthétique comme les techniques de fabrication, mais sur les propriétaires : les élites cholas de El Alto, un groupe social relativement peu accessible aux enquêteurs, mais dont il faut saisir la cohérence des styles de vie, en matière d'économie familiale, d'organisation collective, de financement d'activités publiques (processions, défilés, etc.) ou de décoration architecturale.

Sur ce dernier point, et pour reprendre les recommandations de Marcel Mauss, l'idée est d'analyser les techniques de construction en elles-mêmes, non seulement pour en reconstituer des rituels mais surtout pour mettre au jour des schèmes pratiques de construction et de conception des bâtiments. Une telle entreprise peut ainsi mobiliser des travaux d'histoire andine et d'histoire de l'art, à l'image de l'étude sur les architectures coloniales des Andes réalisée par Gauvin A. Bailey (2010), qui en remarque l'homologie avec les tissus traditionnels (aguayos) par exemple : il montre l'importance des formes géométriques et des bandes de couleur, appelées pallai, renvoyant à l'ordre et à la « civilisation », contre le monde semi-désertique et sauvage des formes animales figurées en contrepoint des motifs géométriques (ратра). Si l'ordonnancement spatial de l'ensemble est d'inspiration européenne, le symbolisme binaire à l'œuvre peut être vu comme caractéristique des cultures andines. L'étude des formes d'architecture émergentes que sont les « chalets » ouvre alors la voie à une autre compréhension de la spécificité des cultures andines contemporaines: à la fois de leur plasticité et de leur résistance par rapport aux éléments extérieurs, qui exprime la recomposition de rapports de domination locaux en contexte de la globalisation.

\section{Bibliographie}

Abercrombie, T. A. (1998) Pathways of memory and poder: Ethnography and history of an Andean community, Madison, University of Wisconsin Press.

Albó X. (1988) Introducción in Albó Xavier (dir.), Raíces de America. El mundo aymara, Madrid, Alianza Editorial/Unesco: 21-50.

Albó X., Preiswerk M. (1991) "El Gran Poder: Fiesta del Aimara Urbano”, América Indígena 51(2-3): 293-352.

Andreoli, E., D’Andrea; L. (2014) La architectura de Freddy Mamani Silvestre, La Paz, Fundacion Cultural Banco Central de Bolivia.

Arnold, D. (1992) La casa de abobes y piedras del Inka: genero, memoria y cosmos en Qaqachaka, in Arnold, D. (dir.) Hacia un orden andino de las cosas. Tres pistas de los Andes meridionales, La Paz, Hisbol/Ilca: 31-108. 
Babadzan, A. (2009) L'indigénisation de la modernité: la permanence culturelle selon Marshall Sahlins, L'Homme, 190, p.105-128.

Bailey G.A. (2010), The Andean Hydrid Baroque. Convergent Cultures in the Churches of Colonial Peru, Notre Dame (Indiana), University of Notre Dame Press.

Bazin, J. (2008) Le chalet basque, in Bazin, J., Des clous dans la Joconde, Toulouse, Anacharsis Editions: 149-162.

Bentmann, R. and M. Müller [1992 (1970)] The Villa as Hegemonic Architecture, London/New Jersey, Humanities Press.

Bishop, R., J. Phillips, W. Yeo (2003) Perpetuating Cities: Exception Globalization and the Southeast Asia Supplement. In R. Bishop, J. Phillips, and W. Yeo (eds.) Postcolonial Urbanism. Southeast Asian Cities and Global Processes, New York/London, Routledge.

Blais, H. (2009) Coloniser l'espace: territoires, identités, spatialité. Genèses, 74, 145-159.

Bourdieu, P. (1967) Postface in Panofsky, E., Architecture gothique et pensée scolastique, Paris, Minuit: 133-167.

Brunskill R.W. (2006 [1985]) Traditional Buildings of Britain: An Introduction to Vernacular Architecture, Londres, Cassels.

Cárdenas, R. (2010) Arquitecturas emergentes en El Alto. El fenómeno estético como integración cultural, La Paz, PIEB.

CDILP (2004) Cámara departamental de industrias de La Paz, Encuesta de necesidades de desarollo empresarial en la industria manufacturera en la ciudad El Alto.

Chadoin, O. (2011) Architecture et pratiques culturelles : une affaire de contexte ?, Communication au colloque « La sociologie de l'architecture : un domaine de savoir en construction ? », Ensa Paris-La Villette, 18 octobre 2011)

Champy F. (2001) Sociologie de l'architecture, Paris, La Découverte.

Crysler, C.G. (2003) Writing Spaces: Discourses of Architecture, Urbanism and the Built Environment, 1960-2000, New York and London, Routledge.

Daly, C. (1872) L'architecture privée au XIX' siècle, vol.2: Villas, chalets, jardins et leurs dépendances diverses, Paris, Ed. Ducher \& Cie.

Darnton R. (2011) Le Grand massacre des chats, Paris, Les Belles Lettres.

Dover, R. (1992) Introduction in Robert V.H. Dover, Katharine E. Seibold \& John H. Mc Dowell, Andean Cosmologies Through Time. Persistence and Emergence, Bloomington, Indiana University Press: 1-16.

Dovey, K. (1996) Framing Places: Mediating Power in Built Form, London \& New York, Routledge.

Garfias, S. \& Mazurek, H. (2005) El Alto desde una perspectiva poblacional, La Paz, CODEPO / IRD.

Gill, L. (2000) Teetering on the Rim, Global Restructuring, Daily Life and the Armed Retreat of the Bolivian State, New York, Columbia University Press.

Guss D. (2008) The Gran Poder and the Reconquest of La Paz, Journal of Latin American Anthropology, 11 (2), p.294-328.

Harris, O. (1995) Ethnic Identity and Market Relations: Indian and Mestizos in the Andes, in Larson, B. \& Harris, O. (dir.), Ethnicity, Markets and Migration in the Andes. At the Crossroads of History and Anthropology, Durham \& London, Duke University Press: 351-390.

Harris, O. (2000) To Make the Earth Bear Fruit: Ethnographic Essays on Fertility, Work and Gender in Highland Bolivia, London, School of Latin American Studies.

Hempele, J. (2008) Arenas of circulation and ethnographic circuits in Hempele, J., Circuits of culture: Media, Politics and Indigenous Identity in the Andes, Minneapolis, University of Minnesota Press: 1-40.

Kearns, G. and C. Philo (1993) Selling Places: The City as Cultural Capital, Past and Present, Oxford, Pergamon Press.

Khol, B. (2004) Privatization Bolivian Style : a Cautionary Tale, International Journal of Urban and Regional Research, 28 (4): 893-908.

King, A. (1984) The Bungalow: The production of a global culture, London/Boston/Melbourne, Routledge \& Kegan.

King, A. (2004) Spaces of Global Cultures: Architecture, Urbanism, Identity, London-New York, Routledge.

Kingman E. G. (ed) (1992) Ciudades de los Andes. Visión histórica y contemporánea, Quito, IFEA. 
Kusno, A. (2000) Behind the Postcolonial: Architecture, Urban Space and Political Culture in Indonesia. London-New York, Routledge.

Lazar, S. (2008) El Alto, Rebel City: Self and Citizenship in Andean Bolivia, Durham, Duke University Press.

Mansilla B.B. (2015) Arquitectura de la complementariedad, La Razon, 11 de Febrero : http://www.larazon.com/opinion/columnistas/Arquitectura-complementariedad_0_2215578471.html

Martinez F. (2010) « Régénérer la race ». Politiques éducatives en Bolivie (1898-1920), Paris, Editions de l'IHEAL.

Mauss, M. (1926) Manuel d'ethnographie, 1926, Consulté en ligne : http://classiques.uqac.ca/classiques/ mauss_marcel/manuel_ethnographie/manuel_ethnographie.html

Mesa C. (2015) Sobre la 'nueva burgesia', Pagina Siete, 08 de Marzo, http://www.paginasiete.bo/ opinion/2015/3/8/sobre-nueva-burguesia-49418.html

Myers, G. (2003) Verandahs of Power: Colonialism and Space in Urban Africa. Syracuse (NY), Syracuse University Press.

Neuman W. (2013) A Colorful Bolivian Bastion, Floatin Above it All, New York Times, May 132013 : http://www.nytimes.com/2013/05/14/world/americas

Orta, A. (2004) Catechizing Culture. Missionnaries, Aymara, and the "New Evangelization". New York, Columbia University Press.

Panofski, E. (1967 [1951]) Architecture gothique et pensée scolastique, Précédé de L'Abbé Suger de Saint-Denis, traduction et postface par Pierre Bourdieu, Paris, Minuit.

Poupeau, F (2010) De la migración rural à la movilidad intra-urbana. Una perspectiva sociológica sobre las desigualdades de acceso al agua en El Alto (Bolivia), in Poupeau, F. and Gonzalez Andricain, C. (eds.). Modelos de gestión del agua en los Andes. Lima, IFEA/PIEB.

Poupeau, F. (2009) El Alto, una ficción política. Bulletin de l'Institut français d'études andines 39 (2): $542-565$.

Poupeau, F. (2011a) The other side of the 'Aymara rebel city': Rural migration, intra-urban mobility and political protest in El Alto, Bolivia. Review of agricultural and environmental studies, 92(4), 417-440.

Poupeau, F. (2011b) L'eau de la Pachamama. Commentaires sur l'idée d'indigénisation de la modernité. L'Homme 198-199: 247-276.

Rudofsky B. (1987 [1964]) Architecture Without Architects: A Short Introduction to Non-Pedigreed Architecture. Albuquerque, University of New Mexico Press.

Sahlins, M. (2007) La Découverte du vrai sauvage et autres essais, Paris, Gallimard, 2007.

Salgueiro, H. (1997) La casaque d'Arlequin. Belo Horizonte, une capitale éclectique au XIX ${ }^{\circ}$ siècle, Paris, Ecole des hautes etudes en sciences sociales.

Socuro Sologuren, X. (2012) La ciudad de los cholos: Mestizaje y colonialidad en Bolivia, siglos XIX y XX, Lima/La Paz, IFEA/PIEB.

Tassi, N. (2010) The Postulate of Abundance: Cholo Market and Religion in La Paz Bolivia. Social Anthropology/Anthropologie Sociale, 18 (2): 191-209.

Teyssot G. (2013) Topology of Everyday Constellations, MIT Press.

UNF (2015) “Cholets. Andean Palaces”, Unitednotion Films, In development.

Vale, L. (1992) Architecture, Power and National Identity, New Haven, Yale University Press.

Vidal, D. (2012) Vivre et travailler entre différents cadres juridiques. Les migrants boliviens dans le secteur de la confection à São Paulo, Revue européenne des migrations internationales, 28 (4): 109-126.

Villanova, R. de \& Garnier, J.-P. (2003) Présentation au dossier Espace et sociétés, n¹13-114, 2003, « Architecture et habitat dans le champ interculturel».

Villanova, R. de (2003) Cultures et architectures de l'entre-deux, Espaces et sociétés, nº113-114: 163-180.

Weber, M. (1982 [1921]) La Ville [Die Stadt], Paris, Aubier.

Weber, M. (2000 [1906]) Les sectes protestantes et l'esprit du capitalisme in Weber M. L'éthique protestante et l'esprit du capitalisme, Paris, Flammarion: 305-342.

Widmarck C. (2003) To Make Do in the City: Social Identities and Cultural Transformations among Aymara Speakers in La Paz, Uppsala, Acta Universitatis Upsaliensis, Studies in Cultural Anthropology, 36. 
Wimmer, A. (2004) Does ethnicity matter? Everyday group formation in three Swiss immigrant neighborhoods. Ethnic and Racial Studies, 27(1): 1-36.

Yeoh, B. (2003 [1996]) Contesting Space: Power Relations and the Urban Built Environment in Colonial Singapore, Oxford, Oxford University Press.

\section{Notes}

1 Le terme aymara désigne un groupe ethnique des hauts plateaux andins (ouest de la Bolivie, sud du Pérou, nord du Chili) qui est considéré comme un «peuple originaire » ayant préexisté non seulement à la colonisation espagnole, mais aussi à l'empire Inca.

2 Du fait du contexte bolivien, on reprend l'idée d'Olivier Chadoin (2011), selon lequel l'architecture est rarement considérée comme une pratique car elle est le plus souvent étudiée du point de vue de l'urbanisme et non de la sociologie.

3 Le travail de conception architecturale n'est pas, dans ce cas, le monopole d'une profession au demeurant peu constituée académiquement et peu reconnue par l'Etat en Bolivie. Sur la profession d'architecte, voir (Champy, 2001, p.6-7).

4 Il est probable que la religion catholique, qui représente encore un héritage colonial fort et structurant sur l'altiplano andin, empêche encore une conversion des élites cholas à l'ascétisme protestant, même dans les versions pentecôtistes qui se sont développées en Amérique latine depuis plusieurs décennies (Orta, 2004).

\section{Pour citer cet article}

\section{Référence électronique}

Franck Poupeau, «Architectures émergentes à El Alto (Bolivie): essai d'interprétation sociologique », Environnement Urbain / Urban Environment [En ligne], Volume 9| 2015, mis en ligne le 19 octobre 2015, consulté le 23 mars 2016. URL : http://eue.revues.org/601

\section{À propos de l'auteur}

\section{Franck Poupeau}

Director, UMI iGLOBES, NRS/University of Arizona, 845 N. Park Avenue, 85719 Tucson (AZ),

USA, franckpoupeau@email.arizona.edu

\section{Droits d'auteur}

(C) Institut National de Recherche Scientifique Centre Urbanisation Culture et Société

\section{Résumé}

This article applies Bourdieu's analysis of the formation of the "scholastic habitus" in medieval times, elaborated in his 1967 afterword to his French translation of Erwin Panofsky's Gothic Architecture and Scholasticism (2005 [1967]), to a different historical case and social context, namely the correspondence between indigenous mental categories and architectural efflorescence in a city of the contemporary Andes: El Alto. This same principle of homology between mental categories and building layout (rooted in a common habitus) can be used to interpret one of the most spectacular characteristics of "emerging architectures" known as chalets in Bolivia. The term chalet designates a hybrid structure consisting of a penthouse and storey dwelling built on the rooftops. The chalets are architectural forms embedded within an economy of symbolic goods characterized by a "dual truth": they are at once material and symbolic; they perform economic functions while at the same time seeking conspicuous exposure and public visibility. The hypothesis developed here is that the conspicuous lifestyle revealed by the construction of chalets on the rooftops can be understood by reference to the emerging social power of the indigenous (cholos) elites. This article thus identifies in 
the fraternities of El Alto the structural equivalent of the scholastic institution that Bourdieu associated with Gothic architecture, in order to reveal the formation of a specific habitus, shared among urban categories defined by similar residential locations, economic activities and forms of collective organization.

\section{Entrées d'index}

Keywords : architecture, dual truth, symbolic power, habitus 